\title{
Distributed Acoustic Sensing Based on Coherent Microwave Photonics Interferometry
}

\author{
Liwei Hua ${ }^{1,2}$, Xuran Zhu ${ }^{1}$ (D) Baokai Cheng ${ }^{1}$, Yang Song ${ }^{1}$, Qi Zhang ${ }^{1}$, Yongji Wu ${ }^{1}$, Lawrence C. Murdoch ${ }^{2}$, \\ Erin R. Dauson ${ }^{3,+}$, Carly M. Donahue ${ }^{3,+}$ and Hai Xiao ${ }^{1, *}$ \\ 1 Holcombe Department of Electrical and Computer Engineering, Clemson University, \\ Clemson, SC 29634, USA; lhua@g.clemson.edu (L.H.); xuranz@g.clemson.edu (X.Z.); \\ baokaic@g.clemson.edu (B.C.); song5@g.clemson.edu (Y.S.); qzhang9@g.clemson.edu (Q.Z.); \\ yongjiw@g.clemson.edu (Y.W.) \\ 2 Department of Environmental Engineering and Earth Sciences, Clemson University, Clemson, SC 29634, USA; \\ lmurdoc@clemson.edu \\ 3 Geophysics Group, Los Alamos National Laboratory, Los Alamos, NM 87545, USA; \\ erindauson@lanl.gov (E.R.D.); cmd@lanl.gov (C.M.D.) \\ * Correspondence: haix@clemson.edu \\ + LAUR number: LA-UR-21-21183.
}

check for updates

Citation: Hua, L.; Zhu, X.; Cheng, B.; Song, Y.; Zhang, Q.; Wu, Y.; Murdoch, L.C.; Dauson, E.R.; Donahue, C.M.;

Xiao, H. Distributed Acoustic Sensing Based on Coherent Microwave Photonics Interferometry. Sensors 2021, 21, 6784. https://doi.org/ $10.3390 /$ s21206784

Academic Editors: Gary R. Pickrell, Brian Risch and Daniel Homa

Received: 11 September 2021

Accepted: 7 October 2021

Published: 13 October 2021

Publisher's Note: MDPI stays neutral with regard to jurisdictional claims in published maps and institutional affiliations.

Copyright: (c) 2021 by the authors. Licensee MDPI, Basel, Switzerland. This article is an open access article distributed under the terms and conditions of the Creative Commons Attribution (CC BY) license (https:// creativecommons.org/licenses/by/ $4.0 /)$.

\begin{abstract}
A microwave photonics method has been developed for measuring distributed acoustic signals. This method uses microwave-modulated low coherence light as a probe to interrogate distributed in-fiber interferometers, which are used to measure acoustic-induced strain. By sweeping the microwave frequency at a constant rate, the acoustic signals are encoded into the complex microwave spectrum. The microwave spectrum is transformed into the joint time-frequency domain and further processed to obtain the distributed acoustic signals. The method is first evaluated using an intrinsic Fabry Perot interferometer (IFPI). Acoustic signals of frequency up to $15.6 \mathrm{kHz}$ were detected. The method was further demonstrated using an array of in-fiber weak reflectors and an external Michelson interferometer. Two piezoceramic cylinders (PCCs) driven at frequencies of $1700 \mathrm{~Hz}$ and $3430 \mathrm{~Hz}$ were used as acoustic sources. The experiment results show that the sensing system can locate multiple acoustic sources. The system resolves $20 \mathrm{n} \varepsilon$ when the spatial resolution is $5 \mathrm{~cm}$. The recovered acoustic signals match the excitation signals in frequency, amplitude, and phase, indicating an excellent potential for distributed acoustic sensing (DAS).
\end{abstract}

Keywords: fiber optics sensors; microwave photonics; interferometry; distributed acoustic sensing (DAS); optical frequency domain reflectometry (OFDR)

\section{Introduction}

Distributed acoustic sensing (DAS) employs optical fiber to acquire many acoustic signals using one interrogation unit. Optical fibers are small, have low loss, and are resistant to corrosion and electromagnetic interference, so they can be easily embedded into structures and function in harsh environments. Thus, DAS has become a powerful tool to understand geodynamics [1,2], and it also provides safety and integrity monitoring solutions in the fields of transportation [3], oil and gas [4], civil infrastructures [5], and related. These applications have diverse requirements in spatial resolution, measurement range, sensing bandwidth, and sensitivity. This has led to two main approaches, one involving DAS measurements in the time domain and another in the frequency domain [6].

The time-domain approaches, including phase-sensitive optical time-domain reflectometry ( $\Phi-$ OTDR) and related [7-9], work by sending light pulses into fiber and collecting the backscattered light in the time domain. They have the advantages of high sensitivity, real-time detection, and long measurement range $[9,10]$. Methods such as data averaging, optical pulse coding [11], coherence detection [12], chirped pulse amplification [13], etc., 
have been developed to increase the signal-to-noise ratio (SNR) and spatial resolution. However, as the light pulse energy is proportional to the pulse width and positively related to the SNR, the time-domain approaches have inherent tradeoffs between spatial resolution, sensing range, measurement accuracy, and measurement time [14]. Typically, the acoustic detection band of $\Phi$-OTDR ranges from tens of kiloherz for a few kilometers to hundreds of herz for more than $100 \mathrm{~km}$ with meter-level spatial resolution [15].

Frequency-domain approaches based on optical frequency-domain reflectometry (OFDR) offer higher spatial resolution without impairing the SNR [16]. In a typical OFDR system, a strong coherent, continuous lightwave is used as the probe. The center wavelength of the light is swept over a certain wavelength band. The spatial resolution is inversely proportional to the frequency sweep range, which can reach the submillimeter scale [17]. Recent developments of OFDR for DAS have focused on increasing the sweep repetition rate [18], decreasing the computational complexity [16,19], and suppressing crosstalk among sensors [20]. A bandwidth of more than a kilohertz was achieved using several different OFDR methods $[18,20]$. However, the measurement range of conventional OFDR systems is significantly shorter than the time-domain methods due to coherence fading and polarization fading. This has led to intricate polarization diversity detection methods, but even with these techniques, the typical measurement range for OFDR DAS is less than several kilometers [20,21].

Microwave photonics technologies have been investigated for distributed sensing in recent years [22-25]. The sensing systems use microwave-modulated light as the probe, and the backscattering from the sensing fiber is recorded in the microwave frequency domain through down conversion. The highly stable microwave phase determines locations along the signal path and thus enables a measurement range of more than $10 \mathrm{~km} \mathrm{[26].}$ Frequency modulation in the gigaherz bandwidth enables spatial resolution in the centimeter range [27]. Distributed systems based on this approach have been developed for interrogating Rayleigh scattering [28], fiber Bragg grating (FBG) arrays [29-31], and interferometers [24,27]. Recently, coherence-length-gated microwave photonics interferometry (CMPI) was developed to improve strain sensitivity using cascaded interferometers [24,32]. The coherence length of the light probe is well controlled to enable localized high-contrast optical interference while avoiding crosstalk among interferometers. The in-fiber ultrafast laser-inscribed weak reflector arrays are used to form the interferometers, which has been demonstrated to significantly reduce interference fading and enhance the SNR in a distributed sensing system [33,34]. However, most of the above-mentioned systems require scanning through the entire microwave band to acquire one frame of distributed strain information, and this limits the measurement bandwidth to less than $100 \mathrm{~Hz}$. Recent investigations show that microwave multitone modulation [35] or sparse frequency measurements [30] can significantly improve the measurement rate, resulting in tens of kilohertz of sensing bandwidth. However, either the sensing range or the number of sensing units is limited by those approaches.

The objective of this paper is to describe a new method for DAS measurements made with CMPI. This method records the strains caused by acoustic signals during the microwave frequency scanning and separates the strain signals in space through a time-frequency joint approach. As a result, an acoustic detection bandwidth of tens of kilohertz for distributed sensing with centimeter-scale spatial resolution can be obtained. The bandwidth has been increased more than a thousand times compared to our former reported microwave photonics distributed sensing systems $[24,27,36]$.

\section{Mathematical Model}

A mathematical model of the CMPI system represents its main components, including a microwave source, a vector microwave detector, a light source, an electro-optical modulator, a photodetector (PD), and the distributed sensors formed by the interferometers (Figure 1a). A continuous-wave (CW) laser with a center frequency of $\omega$ is used as the light source. The light intensity is modulated by a microwave signal. The light launches into an 
optical fiber sensing network and is reflected by reflectors with respective time delays $\tau$. Any two reflections that have a lag $\left(\tau_{k 2}-\tau_{k 1}\right)$ much smaller than the coherence time of the laser produce optical interference, so the responsive light paths form an interferometer $\mathrm{I}_{k}$. Light reflections are injected into a PD, which converts the light power into electric voltage and sends it back to the vector microwave detector. The frequency of the microwave signal is swept over a designed range. At each modulation frequency $\Omega$, synchronized detection is conducted, and the amplitude variation and phase shift of the modulation envelope are measured.
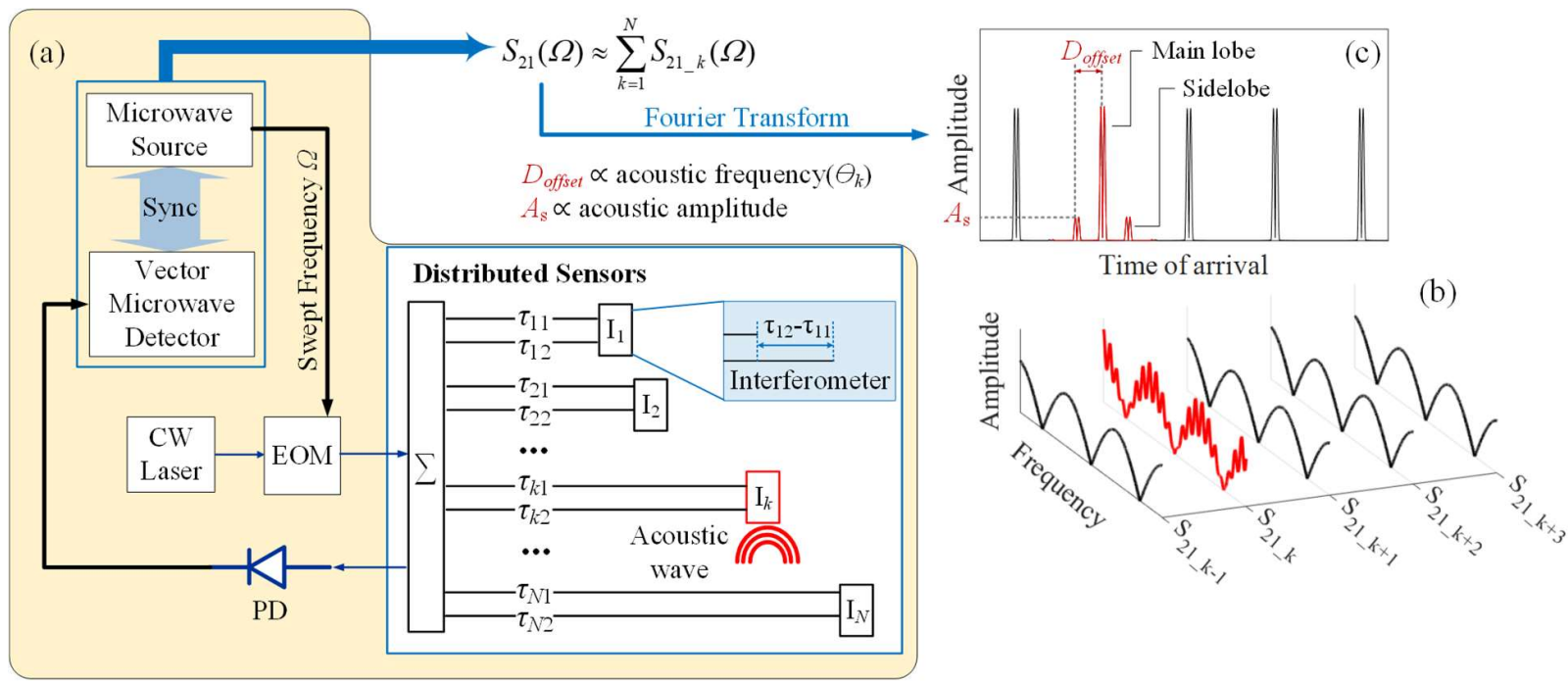

Figure 1. (a) Schematic illustration of the CMPI system for acoustic distributed acoustic sensing. PD: photodetector; EOM: electro-optic modulator; CW laser: continuous-wave laser. (b) The amplitude of the decomposed frequency-domain signal from each interferometer. (c) The amplitude of the time-domain signal.

We designed the optical path difference (OPD) for each interferometer to be much smaller than the coherence length of the light source. The separation distance between adjacent interferometers can also be designed to be much larger than the laser coherence length, so the optical interference only occurs within each interferometer, and it is negligible among different interferometers. The sensing network system is equivalent to a linear combination of independent interferometers $I_{k}(k=1,2, \ldots, N)$. Thus, the complex frequency response of the system $S_{21}$ can be approximately expressed as:

$$
S_{21}(\Omega) \approx \operatorname{rect}\left(\frac{\Omega-\Omega_{c}}{B_{\Omega}}\right) \cdot \sum_{k=1}^{N} S_{21 \_k}(\Omega),
$$

where $B_{\Omega}$ and $\Omega_{c}$ are the bandwidth and center frequency of the microwave signal, respectively, and $S_{21 \_}(\Omega)$ is the frequency response from the $k$ th interferometer, which is expressed as [24,32]:

$$
S_{21 \_k}(\Omega)=m\left[\left(A_{k 1}^{2}+A_{k 1} A_{k 2} \cos \Delta \phi_{k}\right) e^{-\mathrm{j} \tau_{k 1} \Omega}+\left(A_{k 2}{ }^{2}+A_{k 1} A_{k 2} \cos \Delta \phi_{k}\right) e^{-\mathrm{j} \tau_{k 2} \Omega}\right],
$$

where $m$ is a constant decided by the modulation depth of the EOM; $A_{k 1}$ and $A_{k 2}$ are the amplitudes of the two reflected waves; $\Delta \phi_{k}$ is the optical phase difference between the two waves.

If the OPD of the two reflected waves in the $k$ th interferometer $\left(L_{k}\right)$ changes sinusoidally as a function of time, then the optical phase difference varies accordingly and is expressed as:

$$
\Delta \phi_{k}=\left[L_{k}+\delta_{k} \cos \left(\Theta_{k} t+\Phi_{0}\right)\right] \omega / c,
$$


where $\Theta_{k}$ and $\delta_{k}$ are the frequency and amplitude of the dynamic OPD changes, respectively, and $t$ is the time variable.

If the microwave modulation frequency is linearly scanned with a step of $\Delta \Omega$ and a constant sampling time $\Delta t$, we can express $t$ as:

$$
t=\left(\Omega-\Omega_{\text {start }}\right) / \Delta \Omega \cdot \Delta t,
$$

where $\Omega_{\text {start }}$ is the first frequency within the microwave scanning band.

By applying the Fourier-Bessel series, $\cos \Delta \phi_{k}$ can be expressed as:

$$
\cos \Delta \phi_{k}=J_{0}\left(\frac{\delta_{k} \omega}{c}\right) \cos \left(\frac{L_{k} \omega}{c}\right)+2 \sum_{m=1}^{\infty} J_{m}\left(\frac{\delta_{k} \omega}{c}\right) \cdot \cos \left(\frac{L_{k} \omega}{c}+\frac{m \pi}{2}\right) \cos \left[m\left(\frac{\Theta_{k} \Omega}{\Delta \Omega} \Delta t+\Phi_{\text {cons }}\right)\right],
$$

where:

$$
\Phi_{\text {cons }}=\Phi_{0}-\Theta_{k} \Omega_{\text {start }} / \Delta \Omega \cdot \Delta t .
$$

When the amplitude of the dynamic OPD change is much smaller than $2 \pi$ times the wavelength of the light carrier, i.e., $\delta_{k} \ll c / \omega$, Equation (5) can be approximated by keeping the low order $\left(J_{0}\right.$ and $\left.J_{1}\right)$ Bessel terms. The linear approximation is expressed as:

$$
\cos \Delta \phi_{k} \approx \cos \frac{L_{k} \omega}{c}-\frac{\delta_{k} \omega}{c} \sin \frac{L_{k} \omega}{c} \cos \left(\frac{\Theta_{k} \Omega}{\Delta \Omega} \Delta t+\Phi_{\text {cons }}\right)
$$

Substituting Equation (7) into Equation (2), the result indicates that the dynamic OPD change with the frequency $\Theta_{k}$ is modulated on the amplitude of the microwave interferogram $S_{21 \_k}$, as illustrated in Figure 1b. The complex inverse Fourier transform of the $S_{21}(\Omega)$ can be expressed as:

$$
F\left(t_{z}\right)=\left[m B_{\Omega} \sin \mathrm{c}\left(B_{\Omega} t_{z}\right) e^{-j \Omega_{c} t_{z}}\right] * \sum_{k=1}^{N} F_{k}\left(t_{z}\right),
$$

which includes "Static" and "Dynamic" components as:

$$
\begin{gathered}
F_{k}\left(t_{z}\right)=\left\{\left[A_{k 1}{ }^{2}+A_{k 1} A_{k 2} \cos \frac{L_{k} \omega}{c}\right] \delta\left(t_{z}-\tau_{k 1}\right)+\left[A_{k 2}{ }^{2}+A_{k 1} A_{k 2} \cos \frac{L_{k} \omega}{c}\right] \delta\left(t_{z}-\tau_{k 2}\right)\right\}_{\text {Static }} \\
-\left\{\begin{array}{c}
\frac{\delta_{k} \omega}{c} A_{k 1} A_{k 2} \sin \frac{L_{k} \omega}{c} \cdot\left[\delta\left(t_{z}-\tau_{k 1}+\Theta_{k} \frac{\Delta t}{\Delta \Omega}\right) e^{j \Phi_{c o n s}}+\delta\left(t_{z}-\tau_{k 1}-\Theta_{k} \frac{\Delta t}{\Delta \Omega}\right) e^{-j \Phi_{c o n s}}\right] \\
+\frac{\delta_{k} \omega}{c} A_{k 1} A_{k 2} \sin \frac{L_{k} \omega}{c} \cdot\left[\delta\left(t_{z}-\tau_{k 2}+\Theta_{k} \frac{\Delta t}{\Delta \Omega}\right) e^{j \Phi_{\text {cons }}}+\delta\left(t_{z}-\tau_{k 2}-\Theta_{k} \frac{\Delta t}{\Delta \Omega}\right) e^{-j \Phi_{\text {cons }}}\right]
\end{array}\right\}_{\text {Dynamic }}
\end{gathered}
$$

The "Static" component in Equation (9) includes two time-pulses (main lobes) that are generated by the two reflected waves from interferometer $\mathrm{I}_{k}$, and they are centered at and, respectively. The "Dynamic" component is formed by time pulses generated by the dynamic OPD changes. The dynamic pulse pairs behave as sidelobes, which occur at both sides of the respective main lobes (Figure 1c).

The offset between a sidelobe and the corresponding main lobe $\left(D_{\text {offset }}\right)$ is directly proportional to the acoustic frequency $\Theta_{k}$ with a scaling coefficient $\Delta t / \Delta \Omega$. The amplitude of the dynamic OPD change $\delta_{k}$ is directly proportional to the amplitude of the sidelobes.

We calculate $\delta_{k}$ by using the sidelobe amplitude and the interference phase $L_{k} \omega / c$. The interference phase is calculated using the chirp effect of the EOM to perform quadraturephase demodulation [32]. This is done by tuning the EOM bias to add opposite phase shifts of $\pi / 4$ to the optical interference phase, so the peak values of the two main lobes are in quadrature as $A_{k 1}^{2}+A_{k 1} A_{k 2} \cos \left(L_{k} \omega / c+\pi / 4\right)$ and $A_{k 2}{ }^{2}+A_{k 1} A_{k 2} \cos \left(L_{k} \omega / c-\pi / 4\right)$. The standard quadrature-phase demodulation method can be used to calibrate the conic coefficients $\left(A_{k 1}{ }^{2}, A_{k 2}{ }^{2} A_{k 1} A_{k 2}\right.$, and the quadrature error) and calculate the interference phase [37]. 
We extract $S_{21 \_k}(\Omega)$ from $S_{21}(\Omega)$ through an inverse Fourier transform of the gated time-domain signal as:

$$
S_{21 \_k \_g}(\Omega)=\mathcal{F}\left[F\left(t_{z}\right) \cdot g_{k}\left(t_{z}\right)\right] .
$$

where $g_{k}\left(t_{z}\right)$ is a time-domain gate function that selects the time pulses generated by the $k$ th interferometer.

The temporal signal can be reconstructed by applying a high-pass filter to $\left|S_{21 \_k \_g}(\Omega)\right|$. The time is linearly converted from $\Omega$ using Equation (4), and the amplitude is also corrected by using the calculated interference phase.

\section{Experiment}

Several ways to build a sensing network using the CMPI system have been demonstrated [24,37], and we used two of these methods to validate the proposed concept. The first set of experiments used a single pair of in-fiber reflectors to illustrate the signal processing. The next set of experiments used an array of in-fiber reflectors and an external Michelson interferometer (MI) to verify distributed acoustic sensing. The reflectors used in the experiments were fabricated using femtosecond laser micromachining, which created reflectivity from -35 to $-45 \mathrm{~dB}$ [38].

\subsection{System Configuration}

A single longitude mode of the F-P laser (HP81554, Hewlett-Packard, USA) is filtered out by a bandpass filter (BPF1) and used as the light carrier. The selected mode has a center wavelength of $1543 \mathrm{~nm}$ and a coherence length of $6 \mathrm{~cm}$. The light was intensitymodulated by a microwave signal via an EOM (Lucent, X2623Y, Murray Hill, NJ, USA). The modulation signal was generated by a vector network analyzer (VNA, Agilent E8364B, Santa Clara, CA, USA). The bias voltage of the EOM was provided by an external DC power supply. An inline polarization controller was used to optimize the modulation depth of the EOM. The microwave-modulated light output from the EOM was first amplified by an erbium-doped fiber amplifier (EDFA) 1 and then launched into the distributed sensors. Reflected signals from the distributed fiber sensors were amplified by the EDFA2 and filtered by the BPF2. A high-speed PD detected the filtered signal and passed over the converted electrical signal to VNA through port 2.

\subsection{Frequency and Amplitude Reading}

In this experiment, the sensor was formed by two weak reflectors with a separation distance of $1 \mathrm{~cm}$. The fiber sensor was taped on a piezoceramic cylinder (PCC) (dim. $\varnothing 85 \times 32 \mathrm{~mm})$, as shown in Figure 2a,c. The PCC was driven by an arbitrary waveform generator (AWG, Agilent 33120A, Santa Clara, CA, USA) to generate acoustic signals. The VNA was set to have 16,001 sampling points in the microwave band from $1 \mathrm{GHz}$ to $1.1 \mathrm{GHz}$, enabling a $16 \mathrm{~km}$ interrogation range and $1 \mathrm{~m}$ spatial resolution. Therefore, the reflection from the two reflectors was not separable and was shown as a single pulse at the sensor location $(23.6 \mathrm{~m})$ in the time domain. We set the IFBW to $35 \mathrm{kHz}$ to obtain a $30 \mu \mathrm{s}(\Delta t=30 \mu \mathrm{s})$ sampling time at each modulation frequency.

A sinusoid signal with an amplitude of $1 \mathrm{~V}$ was used to drive the PCC. The signal frequency was swept from $12 \mathrm{kHz}$ to $15.6 \mathrm{kHz}$ with a step of $400 \mathrm{~Hz}$. $S_{21}$ was recorded at each step, and time-domain signals were obtained by applying the Fourier transform. Figure $3 \mathrm{a}$ shows the amplitude of the time-domain signals when $12 \mathrm{kHz}$ and $15.6 \mathrm{kHz}$ acoustic signals were generated by the PCC. The main lobe is located at $23.6 \mathrm{~m}$. The acousticwave-generated first-order sidelobes are at $(-5.9272 \mathrm{~km}, 5.9725 \mathrm{~km})$ and $(-7.7124 \mathrm{~km}$, $7.7597 \mathrm{~km}$ ) when $12 \mathrm{kHz}$ and $15.6 \mathrm{kHz}$ acoustic signals were excited, respectively. The offset between the main lobe and both sidelobes $\left(D_{\text {offset-L, }}, D_{\text {offset-R }}\right)$ increased linearly as the acoustic frequency increased, as shown in Figure $3 \mathrm{~b}$. The slope of the linear fitting line is $0.4959 \mathrm{~m} / \mathrm{Hz}$ (a refractive index of 1.452 was used in the calculation), which is the same as the estimated value from Equation (4). The norm of the residual of the linear fitting is $0.1 \mathrm{~mm} / \mathrm{Hz}$. 

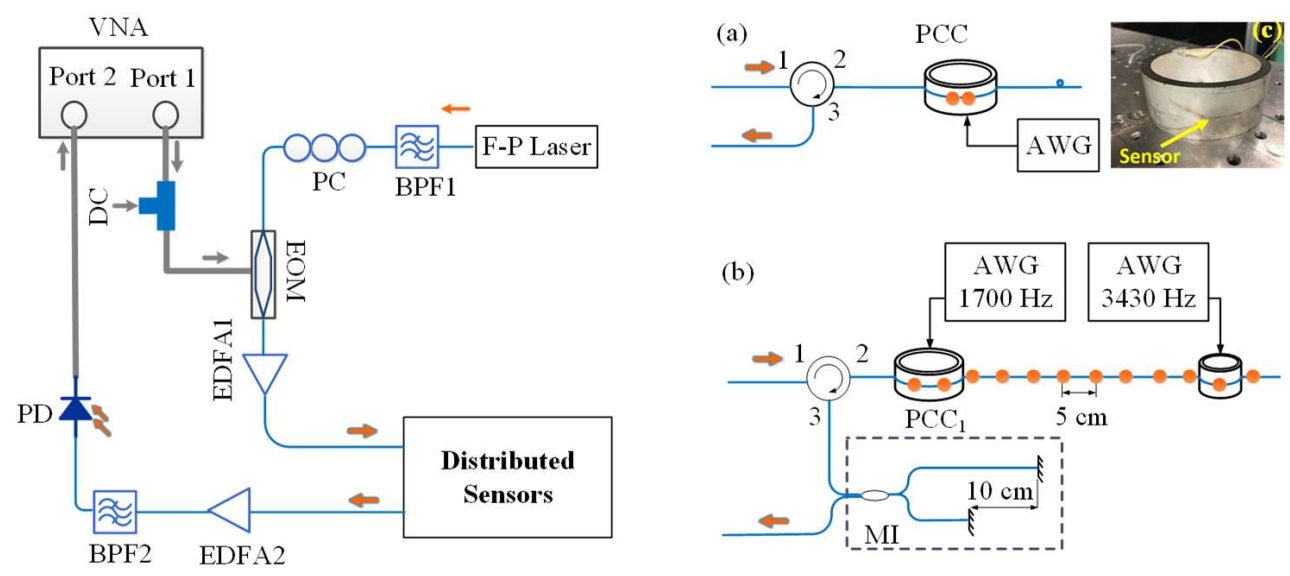

Figure 2. Schematic of the experimental setup for DAS by using CMPI. The optical fiber sensing network was formed by (a) a single reflector pair and (b) a weak reflector array and a Michelson interferometer. (c) Optical fiber with weak reflectors taped around the PCC. PCC: piezo ceramic cylinder; AWG: arbitrary waveform generator, EDFA: erbium-doped fiber amplifier.
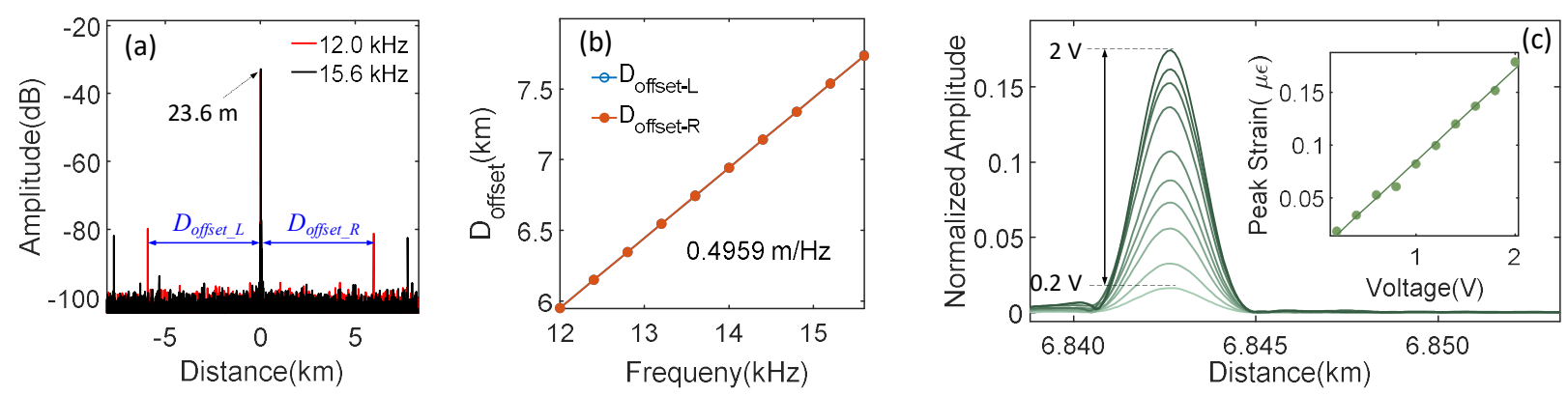

Figure 3. (a) Time-domain signals when $12 \mathrm{kHz}$ and $15.6 \mathrm{kHz}$ acoustic signal are generated by the PCC. (b) The offset between the main lobe and sidelobes as a function of acoustic frequency. (c) Right sideband of the first-order harmonic at the different driving voltages for acoustic frequency of $13.75 \mathrm{kHz}$. Inset: Peak strain as a function of the driving voltage.

The acoustic frequency was then fixed at $13.75 \mathrm{kHz}$, and the amplitude of the acoustic signal was tuned by tuning the driving voltage from $0.2 \mathrm{~V}$ to $2 \mathrm{~V}$ with $0.2 \mathrm{~V}$ per step. The amplitude of the right-sidelobe increased as the driving voltage increased, as shown in Figure $3 \mathrm{~b}$. The amplitudes of the strains $(\varepsilon)$ induced by the acoustic signal were calculated through the peak values of the main lobe and sidelobes. The results show that the peak strain increased linearly as a function of the applied voltage (inset of Figure $3 b$ ).

\subsection{Temporal Signal Reconstruction}

The phase information of the acoustic wave is retained in the time-domain signal, according to Equation (9). Therefore, the proposed method can be used to reconstruct the temporal signal at each sensor location. The reconstruction procedure requires four steps as follows (Figure 4a):

Step 1: Apply a Fourier transform to $S_{21}$ to obtain the time-domain signal.

Step 2: Apply the time-domain gate that is used to filter out the main lobe and sidelobes of the $k$ th sensor.

Step 3: Apply the inverse Fourier transform to the filtered time-domain signal to construct $S_{21-\mathrm{k}}$ for each sensor.

Step 4: Apply the high-pass filter to $\left|S_{21-\mathrm{k}}\right|$ to get the reconstructed temporal signal with normalized amplitude. The actual amplitude of the temporal signal can be calculated by using the peak values of the respective time-domain pulses. 
(a)

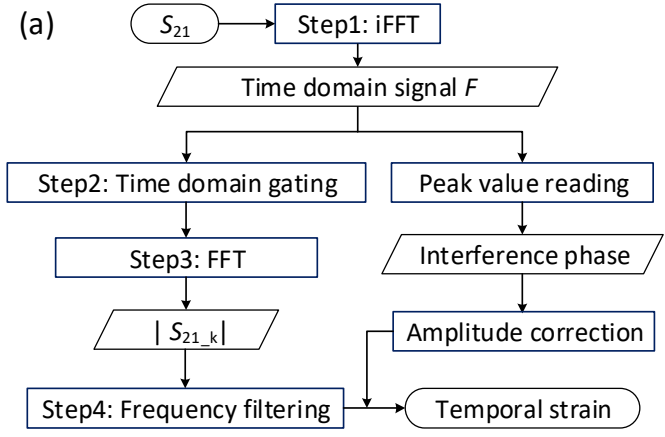

(b)

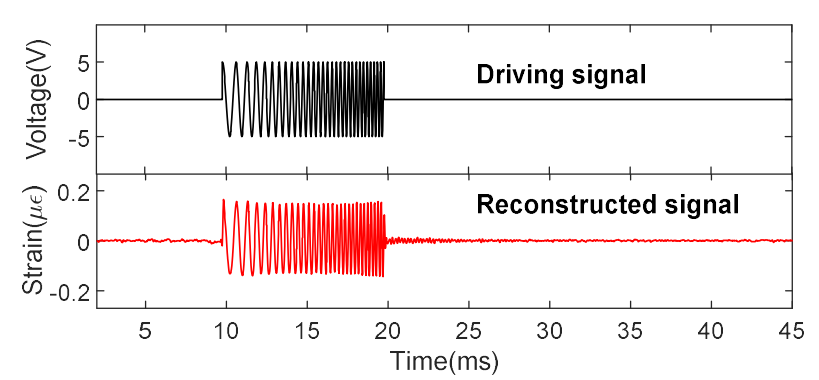

Figure 4. (a) Signal flow chart for temporal signal reconstruction. (b) Driving and reconstructed chirp signals.

We demonstrated the temporal signal reconstruction by using a frequency-chirped pulse. The pulse was triggered by the VNA and applied to the PCC. The pulse width was $10 \mathrm{~ms}$, with a linear variation of the instantaneous frequency from $1 \mathrm{kHz}$ to $5 \mathrm{kHz}$. The separation distance between reflectors was increased from $1 \mathrm{~cm}$ to $15 \mathrm{~cm}$ to increase the strain sensitivity. The F-P laser was substituted by a distributed feedback (DFB) laser (2 MHz linewidth), so the coherence length of the light source was much longer than the OPD of the sensor cavity. The VNA was set to have 1601 sampling points in the microwave band from $1 \mathrm{GHz}$ to $1.3 \mathrm{GHz}$. The test was repeated ten times, and the VNA started scanning $9.781 \mathrm{~ms}$ before the pulse was generated. The temporal signal reconstruction was performed by following the steps shown in Figure 4a. A Tukey window with a cosine fraction of 0.7 was used in Step 2. A second-order Butterworth high-pass filter with a stop frequency of $500 \mathrm{~Hz}$ was used in Step 4.

The driving signal and the averaged reconstructed temporal signal are in phase, and their frequency components are nearly identical (Figure 4b). Amplitudes are also nearly identical using a scaling of $0.03 \mu \varepsilon / \mathrm{V}$. The high-frequency ripples after $20 \mathrm{~ms}$ are only showing in the reconstructed temporal signal, which could be acoustic resonance excited by the acoustic pulse. These results demonstrate that the temporal acoustic signal waveform can be detected by using this method with high fidelity.

\subsection{Distributed Measurement by Using an Array of Reflectors}

Multiple weak reflectors can be cascaded in fiber to create a distributed CMPI sensor. However, dark zones (low-sensitivity regions) need to be inserted between adjacent reflector pairs to avoid the crosstalk among interferometers. One way to avoid dark zones is to fabricate reflectors with similar intervals and use an external Michelson interferometer (MI) in the fiber sensing network [36]. The light is reflected by the reflectors and then coupled into the MI. The coherence length of the light source is much smaller than the intervals of cascaded reflectors. Thus, only the reflector pairs with separation distance close to the arm length difference of the MI $(L)$ are selected out to form the interferometers. The strain changes between selected reflector pairs can be measured using this system.

We used an array of 12 weak reflectors $5 \mathrm{~cm}$ apart on a piece of SMF and an opticalfiber-based MI (Figure 2b) to demonstrate the idea. The MI was made with a $1 \times 2 \mathrm{SMF}$ 50:50 coupler and two Faraday rotation mirrors (FRM). The MI was embedded in a block of epoxy to reduce the OPD change induced by environmental influences (temperature, vibration, etc.). The light was reflected by the reflectors and then reflected by the MI. The coherence length of the light source is shorter than two times the optical interval of the reflectors, i.e., $6 \mathrm{~cm}<2 \cdot n \cdot 5 \mathrm{~cm}$, so only the reflector pairs with separation distance close to the arm length difference of the MI are selected to form the interferometers. For example, the light reflected by the first reflector and longer arm of MI would produce strong interference with the light reflected by the third reflector and shorter arm of MI. The strain changes between first and third reflectors (second and fourth, third and fifth, 
and so on) can be measured using this system. Therefore, 10 sensing units along the SMF were formed for spatially continuous monitoring.

Two piezoceramic cylinders (PCCs) were used to produce acoustic signals at two different locations. A $5 \mathrm{~cm}$ long fiber section centered at $37.05 \mathrm{~m}$ (between the first and second reflector) was taped in the tangential direction along the outer surface of PCC $_{1}$ (dim Ø85 $\times 32 \mathrm{~mm}$ ), and another $5 \mathrm{~cm}$ long fiber section centered at $37.47 \mathrm{~m}$ (around the 11th reflector) was taped along the outer surface of $\mathrm{PCC}_{2}(\operatorname{dim} \varnothing 47 \times 40 \mathrm{~mm})$ as shown in Figure 2. The fiber between the two PCCs was freely placed on an optical table and did not substantially couple acoustic waves. Two AWGs were used to provide driving signals to the PCCs. The driving signals were synchronized and triggered by the VNA. Two driving signals with frequencies of $1700 \mathrm{~Hz}$ and $3430 \mathrm{~Hz}$ were applied to the $\mathrm{PCC}_{1}$ and $\mathrm{PCC}_{2}$, respectively.

The VNA measured the amplitude and phase of the electrical signal at the modulation frequency, which was set to 16,001 sampling points in the microwave band from $0.1 \mathrm{GHz}$ to $15.1 \mathrm{GHz}$. As the sensing range is inversely proportional to the sampling interval, the maximum sensing range was reduced to $80 \mathrm{~m}$. The IFBW was set to $30 \mathrm{kHz}$, so the average sampling time for each sampling point was $0.036 \mathrm{~ms}(\Delta t=0.036 \mathrm{~ms})$. The bandwidth for each sensing section is solely dependent on the IFBW, which was $13.8 \mathrm{kHz}$ for each sensing unit. In the time domain, the pulse reflected by the $k$ th reflector $(k<11)$ and the long arm of the MI were overlapped with the pulse that was reflected by the $(k+2)$ th reflector and the short arm of the MI. Therefore, 14 pulses were formed within the range of $36.93 \mathrm{~m}$ to $37.57 \mathrm{~m}$, as shown in Figure 5a. The peak value of the 3rd, 4th, 11th, and 12th pulses was used to read the interference phase from the interferometers formed by the $(1,3)$, $(2,4),(9,11)$, and $(10,12)$ reflectors, respectively. The four small pulse clusters highlighted by the dashed line boxes in Figure 5 were the sidelobes generated by the vibration from the PCCs. The two pairs of sidelobes located around $30.50 \mathrm{~m}$ (Figure 5b) and $43.61 \mathrm{~m}$ (Figure $5 \mathrm{c}$ ) were symmetrically distributed to the third and fourth pulses, respectively. The two pairs of sidelobes located around $24.18 \mathrm{~m}$ (Figure 5d) and $50.72 \mathrm{~m}$ (Figure 5e) were symmetrically distributed to the 11th and the 12th pulses, respectively. The vibration locations and frequencies were clearly distinguished, as shown in Figure 6a, where the processed strain was plotted as a function of frequency and distance.

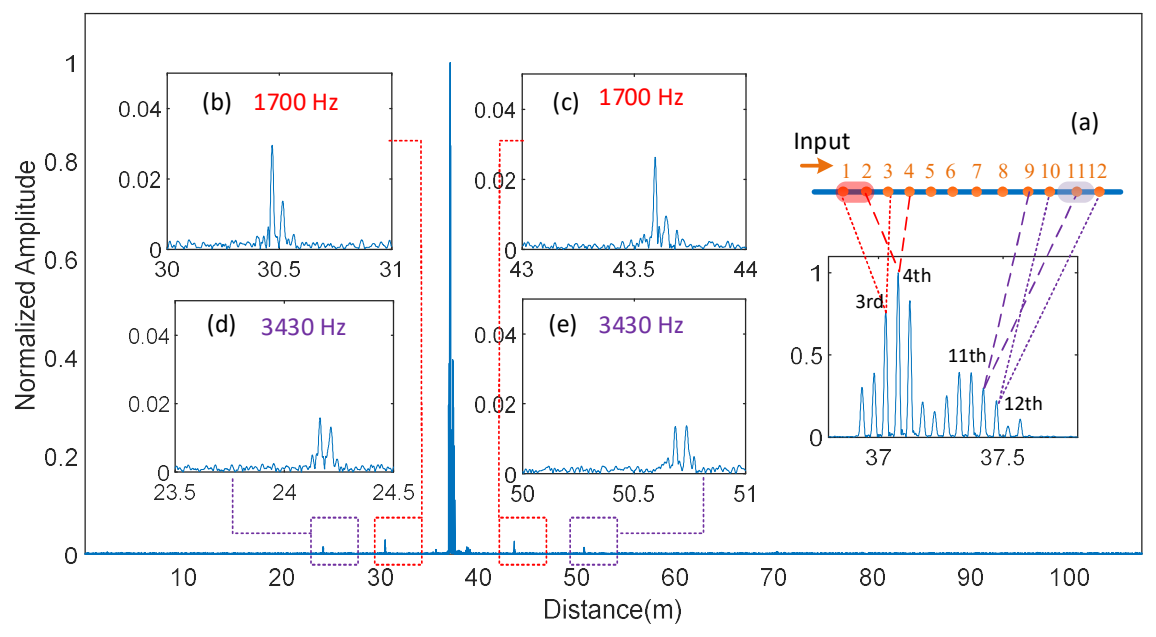

Figure 5. Time-domain signal when the PCCs located at $37.05 \mathrm{~m}$ and $37.47 \mathrm{~m}$ were driven at $1700 \mathrm{~Hz}$ and $3430 \mathrm{~Hz}$. Insets: Expanded time signal. (a) The main lobes formed by the reflector array; $(\mathbf{b}, \mathbf{c})$ sidelobes generated by the PCC1 centered at $37.43 \mathrm{~m}$ and driven at $1700 \mathrm{~Hz} ;(\mathbf{d}, \mathbf{e})$ sidelobes caused by the vibration of PCC2 located at $37.08 \mathrm{~m}$ and driven at $3430 \mathrm{~Hz}$. 
(a)

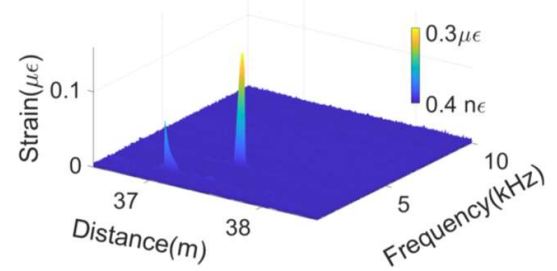

(b)

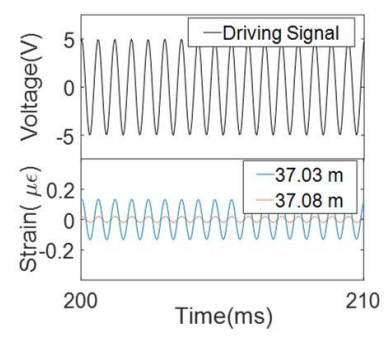

(c)

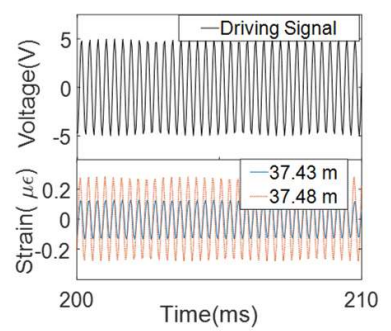

Figure 6. (a) Strain as functions of frequency and distance. (b) Driving signal (upper) to $\mathrm{PCC}_{1}$ and the reconstructed temporal signals centered at $37.03 \mathrm{~m}$ and $37.08 \mathrm{~m}$ (lower). (c) Driving signal (upper) to $\mathrm{PCC}_{2}$ and the reconstructed temporal signals centered at $37.43 \mathrm{~m}$ and $37.48 \mathrm{~m}$ (lower).

The temporal signal reconstruction was performed by following the steps shown in Figure 4a. Time-domain gates that truncated the main pulse and the respective sidelobe at $1700 \mathrm{~Hz}$ and $3430 \mathrm{~Hz}$ were applied to the four affected sensing units separately in Step 2. The reconstructed temporal signals from the sensing units centered at $37.03 \mathrm{~m}$ and $37.08 \mathrm{~m}$ were both in phase with the driving signal to PCC 1 , as shown in Figure $6 \mathrm{~b}$. The reconstructed temporal signals centered at $37.43 \mathrm{~m}$ and $37.48 \mathrm{~m}$ were both in phase with the driving signal to $\mathrm{PCC}_{2}$, as shown in Figure 6c. The strain amplitude was proportional to the contact length between the sensor unit and their respective PCC. The strain amplitude read from the sensor unit centered at $37.03 \mathrm{~m}$ was larger than that at $37.08 \mathrm{~m}$, as the $\mathrm{PCC}_{1}$ was attached to the fiber section from $36.98 \mathrm{~m}$ to $37.03 \mathrm{~m}$. Likewise, the strain amplitude read from the sensor unit centered at $37.48 \mathrm{~m}$ was larger than that at $37.43 \mathrm{~m}$, as more than half of the taped fiber section to $\mathrm{PCC}_{2}$ was after $37.48 \mathrm{~m}$.

The results show that the phase and amplitude of the acoustic signal are well resolved in the reconstructed temporal signals. The amplitude of $19.5 \mathrm{n} \varepsilon$ at $37.08 \mathrm{~m}$ was clearly identified, indicating a lower detection limit, less than that for the experiment setting. The strains from the sensor units that are $5 \mathrm{~cm}$ apart are distinguishable, demonstrating a spatial resolution of $5 \mathrm{~cm}$ for DAS.

Note that there are three terms to describe the range resolution: spatial resolution $\left(S_{R}\right)$, range sampling resolution $\left(S_{r}\right)$, and gauge length $\left(L_{G}\right)$. In this paper, $S_{R}$ refers to the separation distance between the adjacent sensing units; $S_{r}$ refers to the sampling spacing after Fourier transform $\left(S_{r}=c / 2 B_{\Omega} n\right) ; L_{G}$ refers to the arm length difference of the MI. Therefore, in this experiment, the respective values for $S_{R}, S r$, and $L_{G}$ are $5 \mathrm{~mm}, 6.7 \mathrm{~mm}$, and $10 \mathrm{~cm}$, respectively.

\section{Discussion}

\subsection{Maximum Measurable Frequency vs. Reception Bandwidth}

Since the acoustic signal is sampled during microwave frequency scanning, the sampling rate is determined by the frequency scanning sampling time $(\Delta t)$. Therefore, the maximum measurable frequency is expressed as $\Theta_{\max }=1 / 2 \Delta t$. In experiments, we showed that $\Theta_{\max }$ equals $16.6 \mathrm{kHz}$ when the IFBW is set as $35 \mathrm{kHz}$ (Sections 3.2 and 3.3) and $\Theta_{\max }$ equals $13.8 \mathrm{kHz}$ when the IFBW is set as $30 \mathrm{kHz}$ (Section 3.4). An acoustic signal centered at any frequency between DC and $\Theta_{\max }$ could be detected.

However, the sidelobes generated by different sensors may overlap when the acoustic signal bandwidth $\left(B_{a}\right)$ approaches the upper limit, as shown in Figure 7a. These overlaps (spatial aliasing) cause crosstalk among sensors, and more sophisticated signal processing methods are required to identify the frequency components and recover the temporal signal. We call the bandwidth that avoids spatial aliasing the reception bandwidth $\left(B_{r}\right)$, which can be expressed as:

$$
B_{r}=\frac{S_{R}}{2 \gamma}, \text { where } \gamma=\frac{c N \Delta t}{2 B_{\Omega} n} .
$$




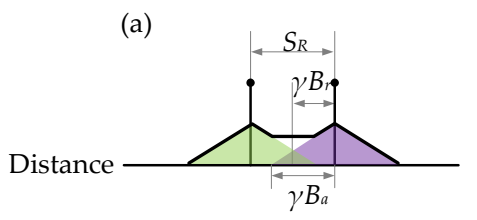

Spatial aliasing $B_{r}<B a$ (b)

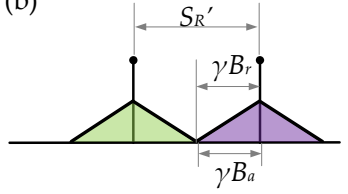

Increase sensors spacing $S_{R}{ }^{\prime}>S_{R}$ (c)

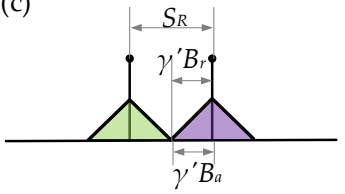

Decrease $\gamma$ by increasing $B \Omega$, or decreasing $N$ or/and $\Delta t . \quad \gamma^{\prime}<\gamma$

Figure 7. Relationship between the spatial resolution $\left(S_{R}\right)$ and reception bandwidth $\left(B_{r}\right)$. (a) Spatial aliasing when $S_{R}<2 \gamma B_{a}$. (b) Increase $S_{R}$ or (c) decrease $\gamma$ to avoid spatial aliasing.

The reception bandwidth can be increased by fabricating the reflectors with larger intervals (Figure $7 \mathrm{~b}$ ), or decreasing $\gamma$ (Figure $7 \mathrm{c}$ ) through increasing the frequency scanning bandwidth $\left(B_{\Omega}\right)$, decreasing the scanning points $(N)$, or reducing the sampling time $(\Delta t)$. These four parameters also affect other aspects of system performance, such as the range sampling resolution $(S r)$, maximum measurement range $\left(L_{r}=c N / 2 B_{\Omega} n\right)$, and signal-tonoise ratio (inversely proportional to $\Delta t$ ). The tradeoffs among the different aspects of system performances can be tuned to satisfy the requirements of different applications.

\subsection{Number of Sensors}

The maximum number of sensing units $N_{C}$ in the system is the ratio of maximum measurement range and the spatial resolution $\left(N_{c}=L_{r} / S_{R}\right)$. Use $N_{c}$ to substitute its expression in Equation (11), and we obtain:

$$
N_{c}=\left(2 B_{r} \Delta t\right)^{-1},
$$

Equation (12) indicates that $\Delta t$, at the given, the larger the reception bandwidth, the smaller the number of sensors can be integrated into the system, and vice versa. For example, in the demonstration experiment in Section 3.4, the $N_{c}$ is about 2144, and the reception bandwidth is $6.5 \mathrm{~Hz}$. When increasing the spacing between the sensing unit to $1 \mathrm{~m}$, the reception bandwidth expands to $130 \mathrm{~Hz}$. Accordingly, the $N_{c}$ reduces to 107 units. The loss from the sensing cable can also limit the $N_{c}$, but this factor is not dominating when over several hertz reception bandwidth is required.

\subsection{Delay in Signal Reading}

There is a time delay between the waveform reading and the occurrence due to the frequency sweeping and signal post-processing. The delay is usually from milliseconds to seconds, which can be minimized by choosing a small number of modulation frequencies.

\section{Conclusions}

This paper presents a microwave photonics method for distributed acoustic sensing. This method encodes acoustic wave-induced strains along the sensing fiber in the microwave spectrum and demodulates them through a time-frequency joint method. The maximum measurable acoustic frequency is determined by the microwave frequency scanning rate and is independent of the spatial resolution and measurement range. The system successfully measured a sinusoidal signal of $16.7 \mathrm{kHz}$ in frequency. The concept was further demonstrated using an optical fiber reflector array and a reference MI. Two PCCs were attached to the reflector array at different locations that were $0.5 \mathrm{~m}$ apart and driven by two sinusoidal voltages with different frequencies of $1700 \mathrm{~Hz}$ and $3430 \mathrm{~Hz}$, respectively. The temporal strain signals read from the sensing fiber were reconstructed. The phase, frequency, and magnitude of the reconstructed temporal signal agree with those of the respective driving signal. The results show that the CMPI system can resolve $20 \mathrm{n} \varepsilon$ at kilohertz range when the gauge length is $10 \mathrm{~cm}$ and spatial resolution is $5 \mathrm{~cm}$, indicating an excellent potential for DAS. 
CMPI-based DAS technology enables flexibly choosing the set of sensing range, range resolution, and reception bandwidth based on the application requirements. Therefore, it has broad application prospects. For instance, the long-range and high-sensitivity capabilities allow its use in seismic sensing and downhole fracture detection. The centimeter-scale resolution capability makes it suitable for applications in the aerospace industry and medical care.

Author Contributions: Conceptualization, L.H.; methodology, L.H., X.Z. and B.C.; software, L.H.; validation, L.H., Y.S., Q.Z. and Y.W.; writing-original draft preparation, L.H.; writing-review and editing, X.Z., L.C.M. and E.R.D.; supervision, H.X.; project administration, L.C.M. and C.M.D. All authors have read and agreed to the published version of the manuscript.

Funding: This work was supported in part by the U.S. Department of Energy National Energy Technology Laboratory Grants DE-FE0028292 and FWP-FE-853-17-FY17.

Institutional Review Board Statement: Not applicable.

Informed Consent Statement: Not applicable.

Data Availability Statement: Not applicable.

Conflicts of Interest: The authors declare no conflict of interest. The funders had no role in the design of the study; in the collection, analyses, or interpretation of data; in the writing of the manuscript, or in the decision to publish the results.

\section{References}

1. Walter, F.; Gräff, D.; Lindner, F.; Paitz, P.; Köpfli, M.; Chmiel, M.; Fichtner, A. Distributed acoustic sensing of microseismic sources and wave propagation in glaciated terrain. Nat. Commun. 2020, 11, 2436. [CrossRef]

2. Dou, S.; Lindsey, N.; Wagner, A.M.; Daley, T.M.; Freifeld, B.; Robertson, M.; Peterson, J.; Ulrich, C.; Martin, E.R.; Ajo-Franklin, J.B. Distributed acoustic sensing for seismic monitoring of the near surface: A traffic-noise interferometry case study. Sci. Rep. 2017, 7, 11620. [CrossRef] [PubMed]

3. Ferguson, R.J.; McDonald, M.A.; Basto, D.J. Take the Eh? train: Distributed acoustic sensing (DAS) of commuter trains in a Canadian City. J. Appl. Geophys. 2020, 183, 104201. [CrossRef]

4. Stajanca, P.; Chruscicki, S.; Homann, T.; Seifert, S.; Schmidt, D.; Habib, A. Detection of leak-induced pipeline vibrations using fiber-Optic distributed acoustic sensing. Sensors 2018, 18, 2841. [CrossRef] [PubMed]

5. Barrias, A.; Casas, J.R.; Villalba, S. A review of distributed optical fiber sensors for civil engineering applications. Sensors 2016, 16, 748. [CrossRef]

6. Hartog, A.H. An Introduction to Distributed Optical Fibre Sensors; CRC Press: Boca Raton, FL, USA, 2017.

7. Fernández-Ruiz, M.R.; Costa, L.; Martins, H.F. Distributed acoustic sensing using chirped-pulse phase-sensitive OTDR technology. Sensors 2019, 19, 4368. [CrossRef]

8. Muanenda, Y.; Faralli, S.; Oton, C.J.; Di Pasquale, F. Dynamic phase extraction in a modulated double-pulse $\phi-O T D R$ sensor using a stable homodyne demodulation in direct detection. Opt. Express 2018, 26, 687-701. [CrossRef]

9. Zhu, T.; He, Q.; Xiao, X.; Bao, X. Modulated pulses based distributed vibration sensing with high frequency response and spatial resolution. Opt. Express 2013, 21, 2953-2963. [CrossRef]

10. Muanenda, Y. Recent advances in distributed acoustic sensing based on phase-sensitive optical time domain reflectometry. J. Sensors 2018, 2018, 3897873. [CrossRef]

11. Wang, Z.; Zhang, B.; Xiong, J.; Fu, Y.; Lin, S.; Jiang, J.; Chen, Y.; Wu, Y.; Meng, Q.; Rao, Y. Distributed acoustic sensing based on pulse-coding phase-sensitive OTDR. IEEE Internet Things J. 2019, 6, 6117-6124. [CrossRef]

12. Wang, Z.; Zhang, L.; Wang, S.; Xue, N.; Peng, F.; Fan, M.; Sun, W.; Qian, X.; Rao, J.; Rao, Y. Coherent $\Phi-O T D R$ based on I/Q demodulation and homodyne detection. Opt. Express 2016, 24, 853-858. [CrossRef] [PubMed]

13. Pastor-Graells, J.; Cortés, L.R.; Fernández-Ruiz, M.R.; Martins, H.; Azaña, J.; Martin-Lopez, S.; Gonzalez-Herraez, M. SNR enhancement in high-resolution phase-sensitive OTDR systems using chirped pulse amplification concepts. Opt. Lett. 2017, 42, 1728-1731. [CrossRef] [PubMed]

14. Shan, Y.; Ji, W.; Wang, Q.; Cao, L.; Wang, F.; Zhang, Y.; Zhang, X. Performance optimization for phase-sensitive OTDR sensing system based on multi-spatial resolution analysis. Sensors 2018, 19, 83. [CrossRef] [PubMed]

15. Zinsou, R.; Liu, X.; Wang, Y.; Zhang, J.; Wang, Y.; Jin, B. Recent progress in the performance enhancement of phase-sensitive OTDR vibration sensing systems. Sensors 2019, 19, 1709. [CrossRef] [PubMed]

16. Ding, Z.; Wang, C.; Liu, K.; Jiang, J.; Yang, D.; Pan, G.; Pu, Z.; Liu, T. Distributed optical fiber sensors based on optical frequency domain reflectometry: A review. Sensors 2018, 18, 1072. [CrossRef]

17. Froggatt, M.; Moore, J. High-spatial-resolution distributed strain measurement in optical fiber with Rayleigh scatter. Appl. Opt. 1998, 37, 1735-1740. [CrossRef] 
18. Leviatan, E.; Eyal, A. High resolution DAS via sinusoidal frequency scan OFDR (SFS-OFDR). Opt. Express 2015, $23,33318-33334$. [CrossRef]

19. Shiloh, L.; Eyal, A. Sinusoidal frequency scan OFDR with fast processing algorithm for distributed acoustic sensing. Opt. Express 2017, 25, 19205-19215. [CrossRef]

20. Li, H.; Liu, Q.; Chen, D.; Deng, Y.; He, Z. High-spatial-resolution fiber-optic distributed acoustic sensor based on $\Phi$-OFDR with enhanced crosstalk suppression. Opt. Lett. 2020, 45, 563. [CrossRef]

21. Arbel, D.; Eyal, A. Dynamic optical frequency domain reflectometry. Opt. Express 2014, 22, 8823-8830. [CrossRef]

22. Hervás, J.; Ricchiuti, A.L.; Li, W.; Zhu, N.H.; Fernández-pousa, C.R. Microwave photonics for optical fiber sensors. IEEE J. Sel. Top. Quantum Electron. 2017, 23, 5602013. [CrossRef]

23. Huang, J.; Lan, X.; Wang, H.; Yuan, L.; Xiao, H. Optical carrier-based microwave interferometers for sensing application. Fiber Opt. Sens. Appl. XI 2014, 9098, 90980H.

24. Hua, L.; Song, Y.; Cheng, B.; Zhu, W.; Zhang, Q.; Xiao, H. Coherence-length-gated distributed optical fiber sensing based on microwave-photonic interferometry. Opt. Express 2017, 25, 31362-31376. [CrossRef]

25. Liehr, S.; Wendt, M.; Krebber, K. Distributed strain measurement in perfluorinated polymer optical fibres using optical frequency domain reflectometry. Meas. Sci. Technol. 2010, 21, 94023. [CrossRef]

26. Clement, J.; Maestre, H.; Torregrosa, G.; Fernández-Pousa, C.R. Incoherent optical frequency-domain reflectometry based on homodyne electro-optic downconversion for fiber-optic sensor interrogation. Sensors 2019, 19, 2075. [CrossRef] [PubMed]

27. Huang, J.; Lan, X.; Luo, M.; Xiao, H. Spatially continuous distributed fiber optic sensing using optical carrier based microwave interferometry. Opt. Express 2014, 22, 18757-18769. [CrossRef] [PubMed]

28. Liehr, S.; Nöther, N.; Krebber, K. Incoherent optical frequency domain reflectometry and distributed strain detection in polymer optical fibers. Meas. Sci. Technol. 2009, 21, 017001. [CrossRef]

29. Ricchiuti, A.L.; Hervas, J.; Barrera, D.; Sales, S.; Capmany, J. Microwave photonics filtering technique for interrogating a very-weak fiber bragg grating cascade sensor. IEEE Photonics J. 2014, 6, 1-10. [CrossRef]

30. Bellido, J.C.; Peralta, J.H.; Madrigal, J.M.; Vicente, H.M.; Penalva, G.T.; Fernandez-Pousa, C.R.; Maicas, S.S.; Hervas, J.; Maestre, H.; Torregrosa, G. Fast incoherent OFDR interrogation of FBG arrays using sparse radio frequency responses. J. Light. Technol. 2018, 36, 4393-4400. [CrossRef]

31. Cheng, R.; Xia, L.; Yan, J.; Zhou, J.; Wen, Y.; Rohollahnejad, J. Radio frequency FBG-based interferometer for remote adaptive strain monitoring. IEEE Photonics Technol. Lett. 2015, 27, 1577-1580. [CrossRef]

32. Hua, L.; Zhu, X.; DeWolf, S.; Lei, J.; Zhang, Q.; Murdoch, L.; Xiao, H. Phase demodulation by frequency chirping in coherence microwave photonic interferometry. IEEE J. Sel. Top. Quantum Electron. 2021, 27, 1-9. [CrossRef]

33. Redding, B.; Murray, M.J.; Donko, A.; Beresna, M.; Masoudi, A.; Brambilla, G. Low-noise distributed acoustic sensing using enhanced backscattering fiber with ultra-low-loss point reflectors. Opt. Express 2020, 28, 14638-14647. [CrossRef]

34. Wu, M.; Li, C.; Fan, X.; Liao, C.; He, Z. Large-scale multiplexed weak reflector array fabricated with a femtosecond laser for a fiber-optic quasi-distributed acoustic sensing system. Opt. Lett. 2020, 45, 3685. [CrossRef]

35. Zhou, D.; Dong, Y.; Yao, J. Truly distributed and ultra-fast microwave photonic fiber-optic sensor. J. Light. Technol. 2020, 38, 4150-4159. [CrossRef]

36. Zhu, X.; Hua, L.; Tang, J.; Murdoch, L.; Xiao, H. Microwave photonic reflectometry for dark-zone free distributed optical fiber sensing. Opt. Lett. 2021, 46, 1173-1176. [CrossRef]

37. Požar, T.; Gregorčič, P.; Možina, J. A precise and wide-dynamic-range displacement-measuring homodyne quadrature laser interferometer. Appl. Phys. B 2011, 105, 575-582. [CrossRef]

38. Cheng, B.; Song, Y.; Hua, L.; Xiao, H. Fabrication and characterization of femtosecond laser induced microwave frequency photonic fiber grating. J. Light. Technol. 2020, 38, 5286-5292. [CrossRef] [PubMed] 